\title{
Association of serum uric acid levels with osteoporosis and bone turnover markers in a Chinese population
}

\author{
Dan-dan YAN ${ }^{1, \#}$, Jie WANG ${ }^{1,2, \#, ~ X u-h o n g ~ H O U}{ }^{1}$, Yu-qian BAO ${ }^{1}$, Zhen-lin ZHANG ${ }^{2, *}$, Cheng $\mathrm{HU}^{1,3, *}$, Wei-ping JIA ${ }^{1}$ \\ ${ }^{1}$ Shanghai Diabetes Institute, Shanghai Key Laboratory of Diabetes Mellitus, Shanghai Clinical Center for Diabetes, Shanghai Jiao \\ Tong University Affiliated Sixth People's Hospital, Shanghai 200233, China; ${ }^{2}$ Department of Osteoporosis, Metabolic Bone Disease \\ and Genetic Research Unit, Shanghai Jiao Tong University Affiliated Sixth People's Hospital, Shanghai 200233, China; ${ }^{3}$ Institute for \\ Metabolic Disease, Fengxian Central Hospital Affiliated to Southern Medical University, Shanghai 201499, China
}

\begin{abstract}
Recent evidence shows that uric acid is protective against some neurological diseases, but can be detrimental in many metabolic and cardiovascular disorders. In this study, we examined the association between serum uric acid levels and bone metabolism in Chinese males and postmenopausal females. A total of 943 males and 4256 postmenopausal females were recruited in Shanghai. The levels of serum uric acid and bone turnover markers (BTMs) were detected along with other biochemical traits. In addition, the fat distribution was calculated through MRI and image analysis software, and bone mineral density (BMD) was determined using dual-energy X-ray absorptiometry. For postmenopausal females, the prevalence of osteoporosis was significantly lower in the hyperuricemia group compared with the normouricemic group $(P=4.65 \mathrm{E}-06)$. In females, serum uric acid level was significantly associated with osteoporosis, with odds ratio $(\mathrm{OR})$ and $95 \%$ confidence interval $(95 \% \mathrm{Cl})$ of 0.844 [0.763; 0.933] $(P=0.0009)$ after adjusting for age, body mass index, HbA1c, lean mass, visceral and subcutaneous fat areas, albumin, 25-hydroxyvitamin D3 [25(OH)D3], and parathyroid hormone (PTH). In females, serum uric acid level was positively correlated with the BMD of the femoral neck $(\beta \pm S E: 0.0463 \pm 0.0161 ; P=0.0042)$, total hip ( $\beta \pm$ SE: 0.0433 $\pm 0.0149 ; P=0.0038)$ and L1-4 ( $\beta \pm S E: 0.0628 \pm 0.0165 ; P=0.0001)$ after further adjusting for age, BMI, HbA1c, lean mass, VFA, SFA, albumin, 25(OH)D3 and PTH. Regarding BTMs, serum uric acid level was negatively correlated with N-terminal procollagen of type I collagen (PINP) in females ( $\beta \pm$ SE: $-0.1311 \pm 0.0508 ; P=0.0100$ ). In summary, our results suggest that uric acid has a protective effect on bone metabolism independent of body composition in Chinese postmenopausal females.
\end{abstract}

Keywords: uric acid; osteoporosis; bone mineral density; bone turnover marker

Acta Pharmacologica Sinica (2018) 39: 626-632; doi: 10.1038/aps.2017.165; published online 14 Dec 2017

\section{Introduction}

With the aging of the population and changes in lifestyle, the average prevalence of osteoporosis in adults has reached 13\% in China ${ }^{[1]}$. With its high prevalence and the related disability, morbidity, mortality and costs of osteoporotic fractures, osteoporosis has become a serious public health problem ${ }^{[2]}$ and has received a great amount of attention globally; therefore, many studies have attempted to identify the risk factors of osteoporosis.

As the end product of purine metabolism, uric acid used to

\footnotetext{
"These authors contributed equally to this work.

${ }^{*}$ To whom correspondence should be addressed.

E-mail alfredhc@sjtu.edu.cn (Cheng HU); zzl2002@medmail.com.cn (Zhen-lin ZHANG)

Received 2017-06-13 Accepted 2017-09-11
}

be recognized as a waste product; in fact, excess serum uric acid can cause gouty arthritis and renal lithiasis. However, recent studies have found that uric acid is protective against some neurological diseases ${ }^{[3]}$ but can be detrimental in many metabolic ${ }^{[4]}$ and cardiovascular ${ }^{[5]}$ disorders. Therefore, the role of uric acid remains an oxidant-antioxidant paradox ${ }^{[6]}$.

Reduced bone mineral density (BMD) is related to a pro-oxidative and pro-inflammatory environment ${ }^{[7]}$. Plasma antioxidant levels are markedly decreased in osteoporotic women ${ }^{[8]}$; therefore, bone loss and osteoporosis are linked to oxidative stress and inflammatory status. Epidemiological evidence has indicated that uric acid is positively correlated with BMD and is protective against osteoporosis ${ }^{[9,10]}$. Considering the link between oxidative stress and bone health, the association between uric acid and osteoporosis is likely based on the antioxidant capacity of uric acid ${ }^{[11]}$. However, some studies have 
found no association of uric acid with osteoporosis ${ }^{[12]}$ or osteoporotic fracture ${ }^{[13]}$. Considering the controversial effects of uric acid on osteoporosis found in previous experimental and clinical studies along with the oxidant-antioxidant paradox of uric acid, the role of uric acid in bone metabolism requires further clarification.

Bone loss occurs during the bone remodeling process, which is reflected by bone turnover markers (BTMs ${ }^{[14]}$. Evaluating the associations between uric acid and BTMs, including osteocalcin, N-terminal procollagen of type I collagen (PINP) and $\beta$-cross-linked C-telopeptide of type I collagen ( $\beta$-CTX), will offer more clues regarding the role of uric acid in bone metabolism.

In addition, uric acid concentration is strongly influenced by age, hormonal factors ${ }^{[15]}$ and body composition ${ }^{[16]}$, which also influence BMD and osteoporosis risk ${ }^{[17,18]}$. Therefore, interference from these confounders may also affect the role of uric acid in osteoporosis.

In this study, we aimed to evaluate the association of uric acid with osteoporosis and BTMs in Chinese males and postmenopausal females separately, correcting for age and multiple indices of adiposity.

\section{Materials and methods Ethics statement}

This study was in accordance with the principles of the Second Revision of the Declaration of Helsinki. It has been approved by the Institutional Review Board of Shanghai Jiao Tong University Affiliated Sixth People's Hospital, and the approval number is 2015-KY-002(T). Every participant provided written informed consent.

\section{Subjects}

Our study recruited 5404 participants from the Nicheng community of the Shanghai area. A similar database has also been used in previous studies ${ }^{[19,20]}$. Patients whose uric acid levels were not tested were excluded. Participants with cancer, hepatic disease, kidney disease, or patients using medications (eg, diphosphonate, glucocorticoids, estrogen, diuretics, and allopurinol) that might influence the bone metabolism or uric acid level were also excluded. Finally, 5199 participants, including 943 males and 4256 postmenopausal females, were retained in this study. Patients with serum uric acid levels greater than $7 \mathrm{mg} / \mathrm{dL}$ for males and $6 \mathrm{mg} / \mathrm{dL}$ for females received a diagnosis of hyperuricemia ${ }^{[21]}$.

\section{Clinical measurements}

Age, height $(\mathrm{m})$ and weight $(\mathrm{kg})$ were recorded for all participants. The body mass index (BMI) was calculated as weight/ height $^{2}$. A type 7600-020 Automated Analyzer (Hitachi, Tokyo, Japan) was used to measure fasting serum triglyceride, total cholesterol, low-density lipoprotein cholesterol, highdensity lipoprotein cholesterol and albumin levels in every participant. HbA1c values were tested by high-performance liquid chromatography with a Bio-Rad Variant II hemoglobin testing system (Bio-Rad Laboratories, Hercules, CA,
USA). The fasting levels of bone metabolism-related markers, including osteocalcin in the form of N-terminal osteocalcin molecules, PINP, $\beta$-CTX, serum 25-hydroxyvitamin D3 [25(OH)D3], and parathyroid hormone (PTH), were measured at the same time point with an automated Roche electrochemiluminescence system (Roche Diagnostics Gmbh, Germany). The intra- and inter-assay coefficients of variation (CVs) were $2.9 \%$ and $4.0 \%$ for osteocalcin, $2.3 \%$ and $2.8 \%$ for PINP, $2.5 \%$ and $3.5 \%$ for $\beta$-CTX, $5.6 \%$ and $8.0 \%$ for $25(\mathrm{OH}) \mathrm{D} 3$, and $1.7 \%$ and $2.9 \%$ for $\mathrm{PTH}$, respectively. Regarding body composition, a BC-420 Tanita Body Composition Analyzer (Tanita, Tokyo, Japan) was used to determine body fat percentage, with each subject standing with barefooted on the analyzer footpads; the impedance between the two feet was measured with an alternating current passing through the lower body. Abdominal visceral fat area (VFA), subcutaneous fat area (SFA), and lean mass were calculated using image analysis software (SLICEOMATIC, version 4.2; Tomovision, Inc, Montreal, QC, Canada) and magnetic resonance imaging (MRI) images acquired at the abdominal level between the fourth and fifth lumbar vertebrae with subjects in the supine position using a 3.0-T MRI system (Achieva; Philips, Best, The Netherlands).

\section{BMD measurement and osteoporosis diagnosis}

The BMD values of the lumber spine (L1-4), femoral neck and total hip were determined by Dual-energy X-ray absorptiometry (DXA, Hologic QDR-2000, Hologic Corporation, Waltham, MA, USA), and the CVs for the DXA measurements at L1-4, the total hip, and the femoral neck were $1.39 \%, 0.7 \%$, and $2.22 \%$, respectively. The T-scores for L1-4, the femoral neck, and the total hip were calculated separately using the following formula: (Measured BMD-Young adult mean BMD)/ Young adult population standard deviation (SD) ${ }^{[22]}$. Osteoporosis was defined as a T-score in any site less than -2.5 SD, and participants with T-scores greater than -2.5 SD in all sites were included as controls ${ }^{[23]}$.

\section{Statistical analysis}

All statistical analyses were performed with SAS 9.2 (SAS Institute, Cary, NC, USA). For continuous variables, normality testing was performed, and variables with skewed distributions were log transformed for further analysis. The mean $\pm S D$ or the median (interquartile range) was used for general description, and differences between groups were determined with the Wilcoxon test. For categorical variables, the number of subjects and proportions were used for general description, and the $x^{2}$ test was used to determine differences between groups. Multivariable logistic regression analysis was performed to assess the effects of uric acid on osteoporosis susceptibility. Multiple linear regression analysis was performed to evaluate the associations of uric acid with BMD and BTMs.

\section{Results}

The clinical characteristics of the study samples are summarized in Table 1. As shown in Figure 1, for females, the preva- 


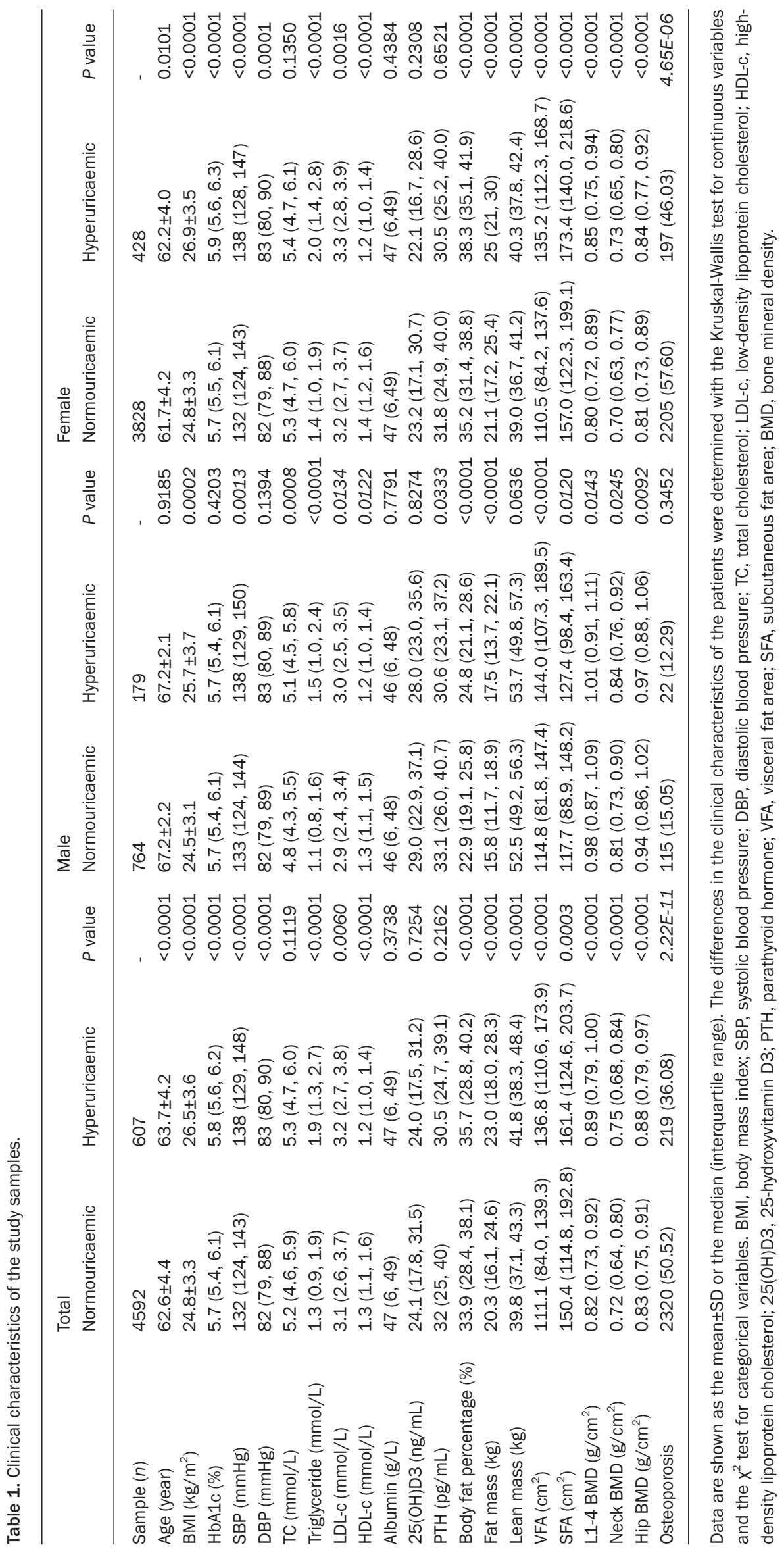




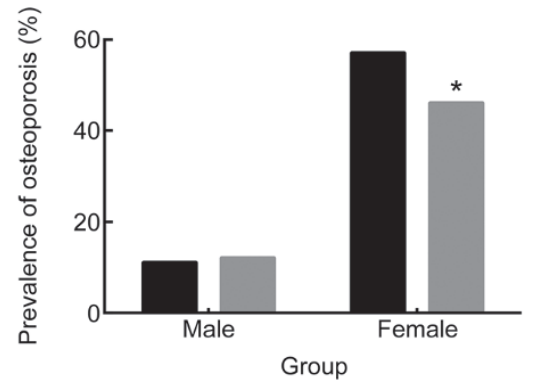

Figure 1. Prevalence rates of osteoporosis in different groups. The prevalence of osteoporosis in the hyperuricemia group was significantly lower than that in the normouricemia group. ${ }^{*} P<0.05$ by the $x^{2}$ test.

lence of osteoporosis was significantly lower in the hyperuricemia group than in the normouricemic group $(P=4.65 \mathrm{E}-06)$. However, no significant distinction was observed for males $(P=0.3452)$. In the multivariable logistic regression analysis, for females, uric acid was significantly associated with osteoporosis after adjusting for age and BMI, with an odds ratio $(\mathrm{OR})$ and $95 \%$ confidence interval $(95 \% \mathrm{CI})$ of 0.874 [0.824;
0.928] ( $P=1.03 \mathrm{E}-05)$, or after further adjusting for age, BMI, HbA1c, lean mass, VFA and SFA (OR [95\% CI]: 0.842 [0.785; 0.904]; $P=2.11 \mathrm{E}-06)$; no similar findings were obtained for males. As serum albumin, 25(OH)D3 and PTH levels can also affect bone metabolism ${ }^{[24]}$, we further evaluated the association between uric acid and osteoporosis adjusted for age, BMI, HbA1c, lean mass, VFA, SFA, albumin, 25(OH)D3 and PTH and found that uric acid was still significantly associated with osteoporosis (OR [95\% CI]: 0.844 [0.763; 0.933]; P=0.0009).

The associations between uric acid and the BMD levels of different sites are shown in Figure 2. When controlling for age, BMI, HbA1c, lean mass, VFA and SFA, uric acid was significantly correlated with the BMD values of the femoral neck and the total hip in both males ( $\beta \pm$ standard error (SE): 0.0462 $\pm 0.0222 ; P=0.0377 ; \beta \pm$ SE: $0.0531 \pm 0.0182 ; P=0.0036)$ and females ( $\beta \pm$ SE: $0.0470 \pm 0.0110 ; P=2.01 \mathrm{E}-05 ; \beta \pm$ SE: $0.0499 \pm 0.0101$; $P=8.65 \mathrm{E}-07)$, but a significant correlation between uric acid and the BMD of L1-4 was only observed in females $(\beta \pm$ SE: $0.0712 \pm 0.0113 ; P=3.01 \mathrm{E}-10)$. Moreover, uric acid was still associated with the BMD of the femoral neck, the total hip and L1-4 in females ( $\beta \pm$ SE: $0.0463 \pm 0.0161 ; P=0.0042 ; \beta \pm$ SE:
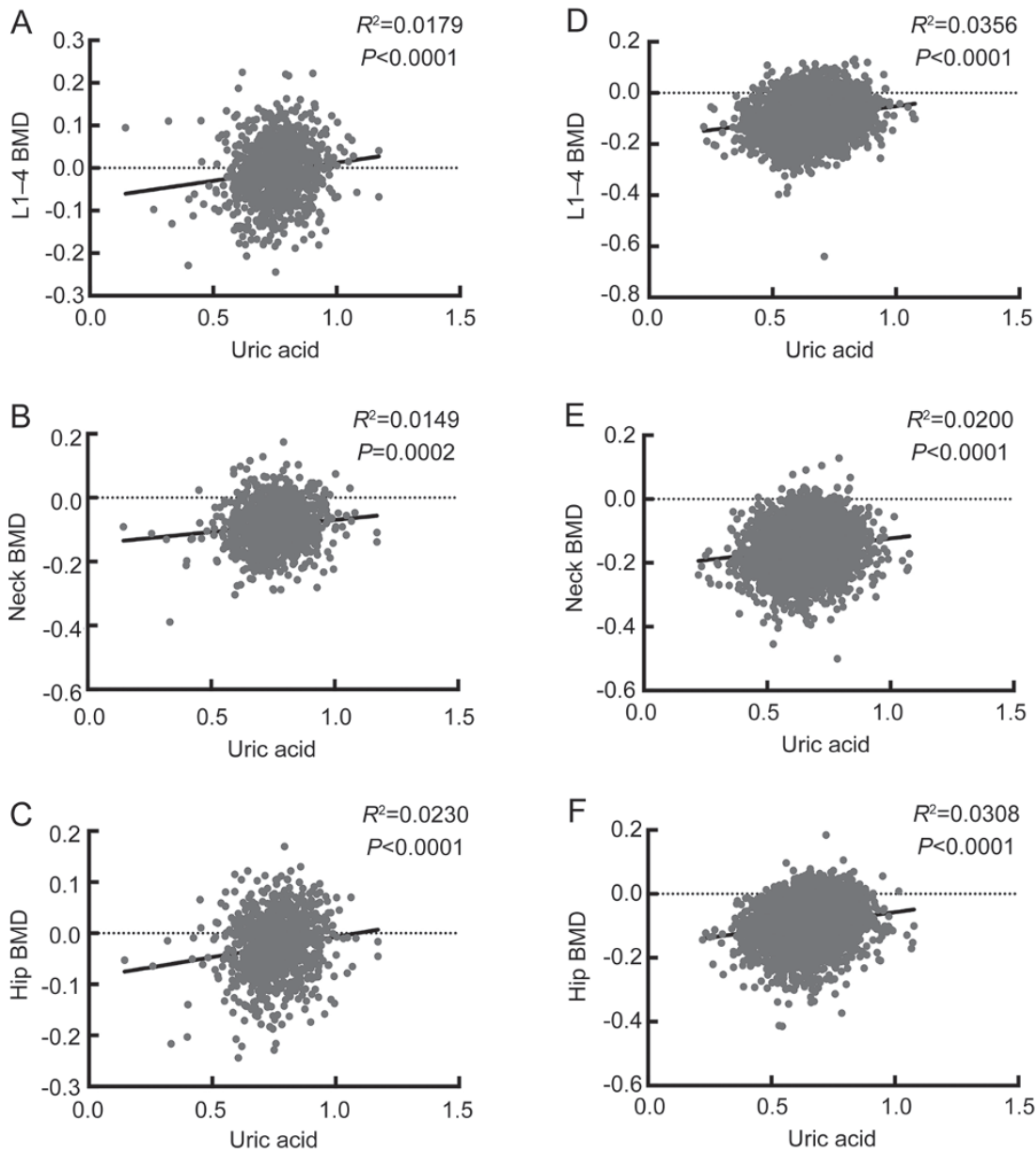

Figure 2. Correlations between uric acid and the BMD levels of different sites. Simple correlations between uric acid and the BMD levels of L1-4, the femoral neck and the total hip were evaluated in males (A, B, C) and postmenopausal females (D, E, F) separately. Uric acid and the BMD levels of L1-4, the femoral neck and the total hip were all analyzed after log transformation. BMD, bone mineral density. 

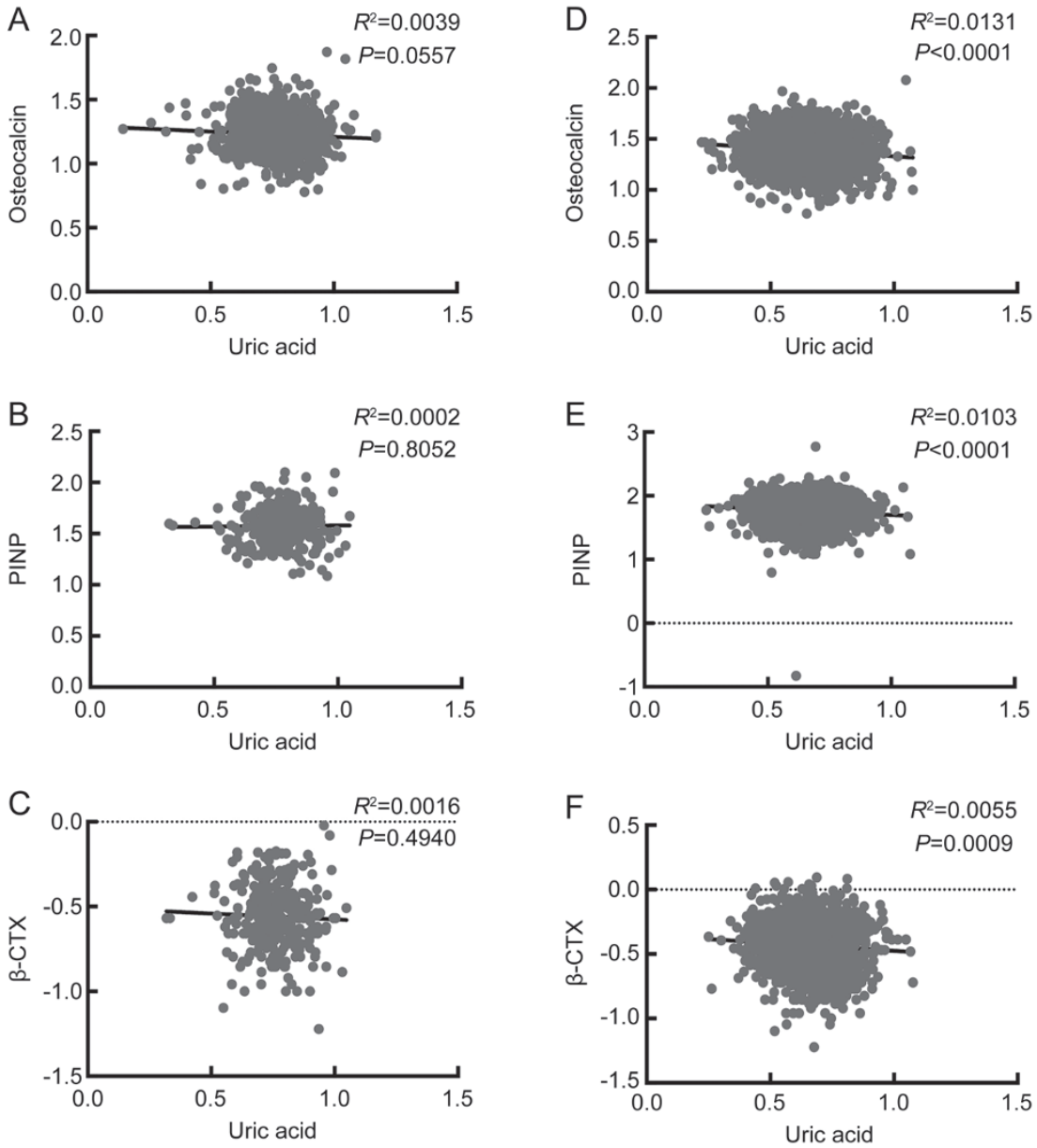

Figure 3. Correlations between uric acid and BTMs. Simple correlations between uric acid and BTMs were evaluated in males (A, B, C) and postmenopausal females (D, E, F) separately. All variables, including uric acid, osteocalcin, PINP and $\beta$-CTX, were analyzed after log transformation. BTM, bone turnover marker; PINP, N-terminal procollagen of type I collagen; $\beta$-CTX, $\beta$-cross-linked C-telopeptide of type I collagen.

$0.0433 \pm 0.0149 ; P=0.0038 ; \beta \pm S E: 0.0628 \pm 0.0165 ; P=0.0001 ;)$ after further adjusting for age, BMI, HbA1c, lean mass, VFA, SFA, albumin, 25(OH)D3 and PTH.

The associations of uric acid with BTMs are shown in Figure 3. When controlling for age and BMI, uric acid was negatively correlated with osteocalcin $(\beta \pm S E$ : $-0.0646 \pm 0.0214 ; P=0.0026)$, PINP $(\beta \pm S E$ : $-0.0146 \pm 0.0424 ; P=0.0006)$ and $\beta$-CTX $(\beta \pm S E$ : $-0.0776 \pm 0.0385 ; P=0.0439)$ in females. However, no similar results were obtained in males. After further adjusting for age, BMI, HbA1c, lean mass, VFA and SFA, uric acid was only negatively correlated with PINP $(\beta \pm S E$ : $-0.1403 \pm 0.0509 ; P=0.0059)$ in females. This association was still significant after further controlling for age, BMI, HbA1c, lean mass, VFA, SFA, albumin, 25(OH)D3 and PTH $(\beta \pm S E$ : $-0.1311 \pm 0.0508 ; P=0.0100)$. No significant associations of uric acid with osteocalcin or $\beta$-CTX were observed in either gender.

\section{Discussion}

As both uric acid and bone metabolism are influenced by age and gender, we mainly focused on the association of uric acid with bone metabolism in males and postmenopausal females separately. In postmenopausal females, uric acid was protec- tive against osteoporosis and was positively correlated with the BMD of L1-4, the femoral neck and the total hip. However, in males, uric acid had no association with osteoporosis risk and was only significantly associated with the BMD of the femoral neck and the total hip. Regarding BTMs, after controlling for age and BMI, uric acid was negatively correlated with osteocalcin, PINP and $\beta$-CTX in postmenopausal females, but no significant associations were obtained in males. Furthermore, after controlling for age, BMI, HbA1c, lean mass, VFA and SFA, uric acid was negatively correlated with PINP in females only.

As hyperuricemia has become an important component of metabolic syndrome, serum uric acid is usually assessed to determine the risk of gouty arthritis or cardiovascular effects; however, the role of uric acid remains controversial, especially in bone health.

In postmenopausal females, uric acid was protective against osteoporosis and was positively correlated with the BMD values of L1-4, the femoral neck and the total hip. Similar to previous observational studies ${ }^{[25,26]}$, our results provide epidemiological evidence that uric acid has a beneficial effect on bone metabolism. The findings of this study are also in 
accordance with functional research showing that uric acid can promote the proliferation and osteogenic differentiation of human mesenchymal stem cells ${ }^{[27]}$. Considering that there is a link between oxidative stress and osteoporosis and that uric acid can function as a central nervous system antioxidant ${ }^{[3]}$, uric acid might exert its protective effects against osteoporosis via its antioxidant function.

PINP, secreted by osteoblasts, is a bone formation marker reflecting collagen formation and osteoblast activation. In this study, uric acid had a negative correlation with PINP independent of age, BMI, HbA1c, lean mass, VFA and SFA in postmenopausal females. During the bone turnover process, bone formation and resorption are tightly coupled, and bone loss tends to occur. Uric acid was negatively correlated with bone formation markers, suggesting that it can reduce the bone turnover rate, thus decreasing bone loss. Regarding other BTMs, uric acid was negatively associated with osteocalcin and $\beta$-CTX after controlling for age and BMI, but this association was attenuated after further controlling for $\mathrm{HbA1c}$, lean mass, VFA and SFA. It has been revealed that reactive oxygen species could mediate osteoclast differentiation ${ }^{[28]}$ and that uric acid, as an an-oxidant, can restrain reactive oxygen; thus, uric acid may reduce bone absorption because of its antioxidant property ${ }^{[29]}$. Furthermore, as several lines of evidence have suggested that body composition and bone metabolism are interrelated $^{[18,30,31]}$, this result indicated that the associations of uric acid with osteocalcin and $\beta$-CTX might due to body composition.

However, uric acid was only associated with the BMD of the femoral neck and the total hip and had no association with osteoporosis susceptibility in males. For bone metabolism, uric acid had no associations with BTMs after controlling for age and BMI or after further controlling for age, BMI, HbA1c, lean mass, VFA and SFA. There are several possible mechanisms that may explain the different roles of uric acid in osteoporosis between the genders. In postmenopausal females, without estrogen protection, uric acid levels will increase more significantly than that in males ${ }^{[32]}$, and the effect of uric acid against bone loss is more obvious ${ }^{[33]}$ than that in males. In addition, the high prevalence of osteoporosis risk factors in males, such as smoking, drinking and eating habits, might neutralize the beneficial effects of uric acid on bone metabolism. Therefore, no significant associations were observed in males.

There are some limitations to our study. First, as the association of uric acid with bone metabolism was only confirmed in our cross-sectional observational study, in which serum uric acid was only tested once, and timely selection errors could not be corrected, further prospective studies or interventional trials are vital to clarify the role of uric acid in osteoporosis. Second, although we controlled for age, blood glucose level and body composition, we did not evaluate lifestyle factors, such as dietary calcium, alcohol consumption, physical activity, smoking, and intake of fruit, tea and vegetables, which might influence bone health ${ }^{[34]}$. Further studies covering such factors will be necessary to evaluate the association between uric acid and bone health. Third, when determining the osteoporosis status of the participants, we did not take fracture status into consideration, although the prevalence rates of osteoporosis fracture were only approximately $12.2 \%$ for males and $14.9 \%$ for females ${ }^{[35]}$; therefore, we could not evaluate the association of uric acid with osteoporotic fracture in the participants. Fourth, we only excluded patients using medications that might influence bone metabolism at the time of investigation; we did not take the medicine history into consideration. Thus, we were unable to exclude the remaining interference of some medicines, such as disphosphonate, glucocorticoids, estrogen, and bisphosphonates, among others, especially bisphosphonates, which have a long half-life.

In conclusion, serum uric acid was protective against osteoporosis, positively correlated with BMD, and negatively associated with bone formation in postmenopausal females. In addition, these effects of uric acid on bone metabolism were independent of obesity and body composition.

\section{Acknowledgements}

We are grateful to the nursing and medical staff at the Shanghai Clinical Center for Diabetes for their help. We would also like to acknowledge all of the study participants.

This work was supported by grants from the National 863 Program (2015AA020110), the Drug Innovation Program of the National Science and Technology Project (2011ZX09307001-02), the Shanghai Young Doctor Training and Funding Program, the Shanghai Jiao Tong Medical/Engineering Foundation (YG2014MS18), the Shanghai Municipal Education Commission-Gaofeng Clinical Medicine Grant Support (20152527), the National Program for Support of Top-notch Young Professionals, the Shanghai Health and Family Planning Commission (2013ZYJB1001), and the Shanghai Hospital Development Center (SHDC12013115).

\section{Author contribution}

Zhen-lin ZHANG and Cheng HU conceived and designed the experiments; Dan-dan YAN and Jie WANG performed the experiments; Dan-dan YAN and Cheng HU analyzed the data; $\mathrm{Xu}$-hong HOU and Yu-qian BAO contributed reagents/materials/analysis tools; Dan-dan YAN and Jie WANG drafted the manuscript; Dan-dan YAN, Jie WANG, Yu-qian BAO, Cheng $\mathrm{HU}$, and Wei-ping JIA wrote the paper. All authors read and approved the final manuscript.

\section{References}

1 Wang Y, Tao Y, Hyman ME, Li J, Chen Y. Osteoporosis in china. Osteoporos Int 2009; 20: 1651-62.

2 Qu B, Ma Y, Yan M, Wu HH, Fan L, Liao DF, et al. The economic burden of fracture patients with osteoporosis in western China. Osteoporos Int 2014; 25: 1853-60.

3 Bowman GL, Shannon J, Frei B, Kaye JA, Quinn JF. Uric acid as a CNS antioxidant. J Alzheimers Dis 2010; 19: 1331-6.

4 Wang T, Bi Y, Xu M, Huang Y, Xu Y, Li X, et al. Serum uric acid associates with the incidence of type 2 diabetes in a prospective cohort of middle-aged and elderly Chinese. Endocrine 2011; 40: 109-16. 
5 Yan D, Wang J, Jiang F, Zhang R, Wang T, Wang S, et al. A causal relationship between uric acid and diabetic macrovascular disease in Chinese type 2 diabetes patients: A Mendelian randomization analysis. Int J Cardiol 2016; 214: 194-9.

6 Sautin YY, Johnson RJ. Uric acid: the oxidant-antioxidant paradox. Nucleosides Nucleotides Nucleic Acids 2008; 27: 608-19.

7 Hardy R, Cooper MS. Bone loss in inflammatory disorders. J Endocrinol 2009; 201: 309-20.

8 Maggio D, Barabani M, Pierandrei M, Polidori MC, Catani M, Mecocci P, et al. Marked decrease in plasma antioxidants in aged osteoporotic women: results of a cross-sectional study. J Clin Endocrinol Metab 2003; 88: 1523-7.

9 Nabipour I, Sambrook PN, Blyth FM, Janu MR, Waite LM, Naganathan V, et al. Serum uric acid is associated with bone health in older men: a cross-sectional population-based study. J Bone Miner Res 2011; 26 : 955-64.

10 Kim BJ, Baek S, Ahn SH, Kim SH, Jo MW, Bae SJ, et al. Higher serum uric acid as a protective factor against incident osteoporotic fractures in Korean men: a longitudinal study using the National Claim Registry. Osteoporos Int 2014; 25: 1837-44.

11 Ishizaka Y, Yamakado M, Toda A, Tani M, Ishizaka N. Relationship between serum uric acid and serum oxidative stress markers in the japanese general population. Nephron Clin Pract 2014; 128: 49-56.

12 Zhang D, Bobulescu IA, Maalouf NM, Adams-Huet B, Poindexter J, Park S, et al. Relationship between serum uric acid and bone mineral density in the general population and in rats with experimental hyperuricemia. J Bone Miner Res 2015; 30: 992-9.

13 Kim SC, Paik JM, Liu J, Curhan GC, Solomon DH. Gout and the risk of non-vertebral fracture. J Bone Miner Res 2017; 32: 230-6.

14 Cabral HW, Andolphi BF, Ferreira BV, Alves DC, Morelato RL, Chambo A Filho, et al. The use of biomarkers in clinical osteoporosis. Rev Assoc Med Bras (1992) 2016; 62: 368-76.

15 Sumino H, Ichikawa S, Kanda T, Nakamura T, Sakamaki T. Reduction of serum uric acid by hormone replacement therapy in postmenopausal women with hyperuricaemia. Lancet 1999; 354: 650.

16 Bonora E, Targher G, Zenere MB, Saggiani F, Cacciatori V, Tosi F, et al. Relationship of uric acid concentration to cardiovascular risk factors in young men. Role of obesity and central fat distribution. The Verona Young Men Atherosclerosis Risk Factors Study. Int J Obes Relat Metab Disord 1996; 20: 975-80.

17 Khosla S, Oursler MJ, Monroe DG. Estrogen and the skeleton. Trends Endocrinol Metab 2012; 23: 576-81.

18 Reid IR. Relationships between fat and bone. Osteoporos Int 2008; 19: 595-606.

19 Wang J, Yan D, Hou X, Chen P, Sun Q, Bao Y, et al. Association of adiposity indices with bone density and bone turnover in the Chinese population. Osteoporos Int 2017; 28: 2645-52.

20 Wang J, Yan DD, Hou XH, Bao YQ, Hu C, Zhang ZL, et al. Association of bone turnover markers with glucose metabolism in Chinese population. Acta Pharmacol Sin 2017; 38: 1611-7.

21 Bardin T, Richette P. Definition of hyperuricemia and gouty conditions. Curr Opin Rheumatol 2014; 26: 186-91.

22 Blake GM, Fogelman I. The role of DXA bone density scans in the diagnosis and treatment of osteoporosis. Postgrad Med J 2007; 83: 509-17.

23 Rossini M, Adami S, Bertoldo F, Diacinti D, Gatti D, Giannini S, et al. Guidelines for the diagnosis, prevention and management of osteoporosis. Reumatismo 2016; 68: 1-39.

24 Hui JY, Choi JW, Mount DB, Zhu Y, Zhang Y, Choi HK. The independent association between parathyroid hormone levels and hyperuricemia: $a$ national population study. Arthritis Res Ther 2012; 14: R56.

25 Ahn SH, Lee SH, Kim BJ, Lim KH, Bae SJ, Kim EH, et al. Higher serum uric acid is associated with higher bone mass, lower bone turnover, and lower prevalence of vertebral fracture in healthy postmenopausal women. Osteoporos Int 2013; 24: 2961-70.

26 Makovey J, Macara M, Chen JS, Hayward CS, March L, Seibel MJ, et al. Serum uric acid plays a protective role for bone loss in peri- and postmenopausal women: a longitudinal study. Bone 2013; 52: 4006.

27 Li HZ, Chen Z, Hou CL, Tang YX, Wang F, Fu QG. Uric acid promotes osteogenic differentiation and inhibits adipogenic differentiation of human bone mesenchymal stem cells. J Biochem Mol Toxicol 2015; 29: 382-7.

28 Lee NK, Choi YG, Baik JY, Han SY, Jeong DW, Bae YS, et al. A crucial role for reactive oxygen species in RANKL-induced osteoclast differentiation. Blood 2005; 106: 852-9.

29 Zhao DD, Jiao PL, Yu JJ, Wang XJ, Zhao L, Xuan Y, et al. Higher serum uric acid is associated with higher bone mineral density in Chinese men with type 2 diabetes mellitus. Int J Endocrinol 2016; 2016: 2528956.

30 Afghani A, Goran MI. The interrelationships between abdominal adiposity, leptin and bone mineral content in overweight Latino children. Horm Res 2009; 72: 82-7.

31 Sotunde OF, Kruger HS, Wright HH, Havemann-NeI L, Kruger IM, Wentzel-Viljoen $\mathrm{E}$, et al. Lean mass appears to be more strongly associated with bone health than fat mass in urban black south African women. J Nutr Health Aging 2015; 19: 628-36.

32 Hak AE, Choi HK. Menopause, postmenopausal hormone use and serum uric acid levels in US women--the Third National Health and Nutrition Examination Survey. Arthritis Res Ther 2008; 10: R116.

33 Garnero P, Sornay-Rendu E, Chapuy MC, Delmas PD. Increased bone turnover in late postmenopausal women is a major determinant of osteoporosis. J Bone Miner Res 1996; 11: 337-49.

34 Adler RA. Risk factors for osteoporosis 2000-2012. Endocrine 2017; 55: 664-5.

35 Liu JM, Ma LY, Bi YF, Xu Y, Huang Y, Xu M, et al. A population-based study examining calcaneus quantitative ultrasound and its optimal cut-points to discriminate osteoporotic fractures among 9352 Chinese women and men. J Clin Endocrinol Metab 2012; 97: 800-9. 